\title{
Microencapsulation of self-healing agents containing a fluorescent dye
}

\author{
H. H. Noh, J. K. Lee \\ Department of Polymer Science and Engineering, Kumoh National Institute of Technology, \#1 Yangho-Dong, Gumi-City, \\ Gyungbuk 730-701, Korea
}

Received 11 June 2012; accepted in revised form 3 September 2012

\begin{abstract}
Two different self-healing agent candidates, endo-dicyclopentadiene (endo-DCPD) and 5-ethylidene-2-norbornene (ENB), containing a fluorescent dye surrounded by a melamine-urea-formaldehyde (MUF) shell were microencapsulated by in-situ polymerization and the resulting microcapsules were characterized in this work. The microcapsules showed a narrow size distribution with a spherical shape and rough outer and smooth inner surfaces for both healing agent systems. Shell thicknesses of the microcapsules were $\sim 880 \pm 80 \mathrm{~nm}$ for endo-DCPD and $\sim 620 \pm 60 \mathrm{~nm}$ for ENB. The incorporation of a fluorescent dye as tracer into self-healing agents did not disturb the formation of microcapsules. The release of self-healing liquid into the induced crack from ruptured microcapsules in an epoxy coating layer was observed using a fluorescence microscopy. The use of a fluorescent dye is very effective in the observation of a damage site.
\end{abstract}

Keywords: coatings, self-healing, microencapsulation, fluorescent dye

\section{Introduction}

A lot of research on self-healing technology is underway for microcapsule-based coating systems over the last decade [1-6]. In this technique, microcapsules rupture upon damage inducing cracks in the coating layer, releasing their encapsulated liquid healing agent into the crack planes. The organic and inorganic self-healing materials, surrounded by urea-formaldehyde (UF) thermosetting shell were microencapsulated, and the microcapsules were embedded into different polymer coating materials, showing protective ability on steel plate (e.g., effective inhibition from metal corrosion and rusting) [1, 2]. A norbornene-based ring opening metathesis polymerization (ROMP) healing agent, endo-dicyclopentadiene (endo-DCPD), as self healing agent was also microencapsulated with UF shell [7-12]. UF capsules filled with endo-DCPD were devel- oped in nano-size, which is important in fabricating thin coating applications [3].

Recently, self-healing agents, 5-ethylidene-2-norbornene (ENB) and ENB with crosslinkers, showing a faster ROMP rate at lower catalyst loadings, were microencapsulated with a ternary melamineurea-formaldehyde (MUF) shell in this laboratory $[11,12]$. The fabrication process is relatively very simple with no external control of $\mathrm{pH}$, and produced a narrow size distribution of capsules with no debris. These microcapsules also had a rough outer surface and exhibited significantly higher thermal stability and less core material permeability when compared to UF capsules. Small changes in the core material during encapsulation process (e.g., going from one core material to a closely related core material), the manufacturing procedure (e.g., order of input), the ratio of the reactants and additives (e.g., typically the ratio between the main compo-

\footnotetext{
${ }^{*}$ Corresponding author, e-mail: jklee@kumoh.ac.kr (C) BME-PT
} 
nents of shell forming reagents), and the reaction conditions (e.g., reacting temperature and time) often lead to significant changes in the formation of acceptable microcapsules.

One of the key aspects for achieving a successful microcapsule-based self-healing system lies with production of adequate microcapsules to be embedded. Thus, microcapsules must be carefully engineered in order to possess requisite strength during handling and processing, long shelf life during storage, and excellent adhesion with the cured polymer matrix [13]. It is also critical that the liquid healing agent within microcapsules is effectively delivered into the crack planes after damage in order to provide efficient recovery of properties. Therefore, it is beneficial to track the release of healing agent through invisible cracks from incorporated microcapsules for the development of self-healing. The addition of tracers such as X-ray dye [14] and UV fluorescent material [15] for hollow fiber reinforced composites and red dye [1] for microcapsule containing coatings into the self-healing agents have been reported for visual observations of damages.

In this study, microcapsules were synthesized for two different self-healing agents, endo-DCPD and ENB, containing a fluorescent dye surrounded by MUF shell. A particle size analyzer (PSA), a thermogravimetric analyzer (TGA) and a scanning electron microscope (SEM) were used to investigate particle size/size distribution and thermal resistance of the microcapsules and to observe morphology of the capsules, respectively. The MUF microcapsules containing a fluorescent dye were dispersed in an epoxy coating layer and a fluorescence microscope (FM) was used to track the transport of liquid healing agent through cracks after inducing the damage on the epoxy coating.

\section{Experimental}

\subsection{Preparation of microcapsules and coatings}

Two healing agent candidates, endo-DCPD (with 95\% endo-isomer, Acros Chemical Co., Belgium) and ENB (Sigma-Aldrich, USA) as core material, were microencapsulated by in-situ polymerization of melamine (M) (Sigma-Aldrich, USA), urea (U) (Sigma-Aldrich, USA) and formaldehyde solution, $37 \mathrm{wt} \%$ in $\mathrm{H}_{2} \mathrm{O}(\mathrm{F})$ (Sigma-Aldrich, USA) to produce the MUF polymer shell in an aqueous solution. Sodium lauryl sulfate (SLS, Junsei, Japan)

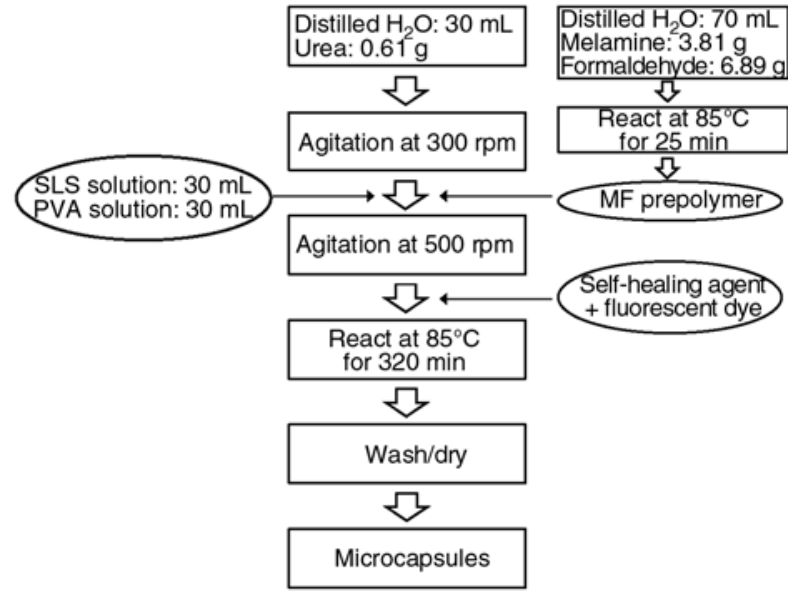

Figure 1. Microencapsulation process

was used as emulsifier, and poly(vinyl alcohol) (PVA, degree of polymerization $=500$, degree of hydrolysis $=99.0 \mathrm{~mol} \%$, Junsei, Japan) as stabilizer. Figure 1 shows the microencapsulation process in this work. A fluorescent dye (derivative of 4,4'diamino-2,2'-stilbenedisulfonic acid, Hwasung Chemcial Co., Ltd., Korea) of $0.05 \mathrm{~g} / \mathrm{L}$ was dissolved in self-healing agents at room temperature for $10 \mathrm{~min}$ before encapsulation. Also, SLS (0.5 wt $\%)$ and PVA (6.3 wt $\%)$ aqueous solutions were prepared by heating at $70^{\circ} \mathrm{C}$ for $20 \mathrm{~min}$ and $2 \mathrm{~h}$, respectively. MF prepolymer solution was obtained from a mixture of $3.81 \mathrm{~g}$ melamine and $6.89 \mathrm{~g} 37 \mathrm{wt} \%$ formaldehyde aqueous solution with $70 \mathrm{~mL}$ distilled water by heating at $70^{\circ} \mathrm{C}$ for $25 \mathrm{~min}$ until it becomes clear. Urea $(0.61 \mathrm{~g})$ was dissolved in $30 \mathrm{~mL}$ distilled water at $\mathrm{RT}$ in a $250 \mathrm{~mL}$ reaction beaker. Subsequently, the MF solution, $30 \mathrm{~mL}$ SLS solution, and $30 \mathrm{~mL}$ PVA solution were added into the reaction beaker, after raising the agitating speed to $300 \mathrm{rpm}$. Prior to slowly adding $30 \mathrm{~mL}$ of core healing agent (endoDCPD or ENB + fluorescent dye) into the beaker, the agitation speed was increased to $500 \mathrm{rpm}$, leading to the formation of small droplets of the core materials. This agitation step was allowed to continue for 10 min at RT to generate the stabilized emulsion, before the temperature was raised to the nominal reaction temperature of $85^{\circ} \mathrm{C}$ for $40 \mathrm{~min}$ and maintained for 320 min under continuous agitation. The microcapsule slurry formed after the isothermal reaction was decanted on filter paper (Advantec no. 2, Toyo Roshi Kaisha Ltd., Japan). The microcapsules were separated into individual capsules easily by hand-shaking after rinsing and drying.

The microcapsules produced were mechanically dispersed into diglydcidyl ether of bisphenol-A 
Table 1. Chemicals used for microencapsulation and epoxy coatings

Thermosetting system
(matrix) Matrix resin

(DGEBA, equivalent weight $=188 \mathrm{~g} \cdot \mathrm{eq}^{-1}$, Kukdo Chem., Korea) epoxy coatings cured with diethylenetriamine (DETA, equivalent weight $=190 \mathrm{~g} \cdot \mathrm{eq}^{-1}$, Kukdo Chem., Korea) at room temperature for 24 hours. Table 1 has chemicals used for microencapsulation and epoxy coatings.

\subsection{Characterization of microcapsules}

The thermal stability of the microcapsules was examined with a thermogravimetric analyzer (TGA, Auto-TGA Q500, TA Instruments, USA) upon heating from room temperature (RT) to $500^{\circ} \mathrm{C}$ for ENBand $600^{\circ} \mathrm{C}$ for endo-DCPD-microcapsules at a scanning rate of $10^{\circ} \mathrm{C} / \mathrm{min}$ in a nitrogen atmosphere. The size and size distribution of the microcapsules were obtained with a particle size analyzer (PSA, Mastersizer 2000, Malvern Instrument, UK). The capsule surface and shell wall thickness were analyzed using a scanning electronic microscope (SEM, JSM-6380, Jeol, Japan) after the microcapsules were spread on an adhesive tape, punctured using a razor blade, and heated on a hotplate at $150^{\circ} \mathrm{C}$ for 12 hours to ensure that the core material completely evaporated.

\subsection{Damage observations}

DGEBA epoxy resin was mixed with $5 \mathrm{wt} \%$ of Grubbs catalyst using a mechanical stirrer for $10 \mathrm{~min}$ at $500 \mathrm{rpm}$, followed by adding DETA curing agent in an equivalent weight and mixing for additional $10 \mathrm{~min} .5 \mathrm{wt} \%$ of microcapsules was added to the epoxy solution and mixed at a slower stirring speed of $150 \mathrm{rpm}$ for $10 \mathrm{~min}$. Air bubbles were removed under vacuum for $10 \mathrm{~min}$. The mixed epoxy resin was then poured into a mold $(50 \mathrm{~mm} \times 5.3 \mathrm{~mm} \times$ $0.2 \mathrm{~mm}$ ) made by polyurethane, and then cured at RT for $24 \mathrm{~h}$ and at $50^{\circ} \mathrm{C}$ for $4 \mathrm{~h}$. A fluorescent microscope (Axiovert 40 CFL, Zeiss, Germany) was employed to observe microcapsules before damage and the transport of healing agent through cracks after damage to the cured coating layer given by hand. The excitation wavelengths used for the observation in this work were $\lambda=350,480$, and $546 \mathrm{~nm}$.

\section{Results and discussion}

\subsection{Thermogravimetric analysis}

Figure 2 shows the weight loss curves of endoDCPD- and ENB-microcapsules containing a fluorescent dye and the MUF capsule shell without the core material. As shown in the figure, there are gradual and similar decreases down to $\sim 70 \%$ in weight for both endo-DCPD- and ENB-microcapsules up to $\sim 300^{\circ} \mathrm{C}$ above which there are sudden weight drops for ENB-microcapsules as reported in the previous work [11]. For endo-DCPD-microcapsules, the sudden drop occurred at $\sim 430^{\circ} \mathrm{C}$, much higher than that of ENB-microcapsules. The dramatic weight loss in a particular temperature of the microcapsules is due to a sudden release of the healing agent. As the temperature increases, the microcapsules become weaker and burst beyond the critical level at a certain temperature by increasing 


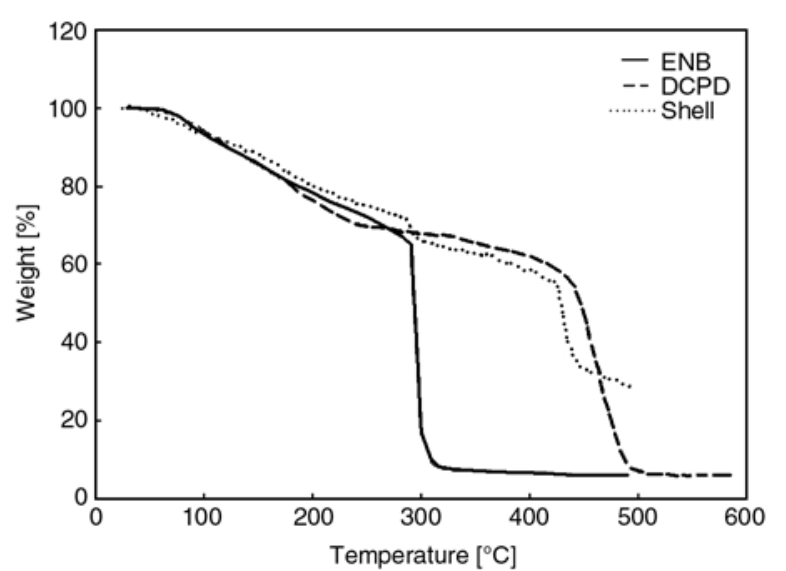

Figure 2. TGA thermogram of endo-DCPD- and ENBmicrocapsules

the internal pressure of the microcapsules and the collapse of the weaken shells [11]. Notice the sudden loss of MUF shell mass at $\sim 420^{\circ} \mathrm{C}$, indicating thermal degradation, which would lead to a dramatic decrease in mechanical strength of the shell. The gradual weight loss below the temperature at which the sudden weight loss takes place may be attributed to the diffusion and evaporation of selfhealing agent through walls of microcapsules. The thicker capsule shell thickness of endo-DCPDmicrocapsules leads to the dramatic weight loss temperature higher than ENB-microcapsules, as will be shown in the SEM image.

\subsection{Particle size analysis}

Figure 3 shows the distribution of the microcapsules using a particle size analyzer. Average diame-

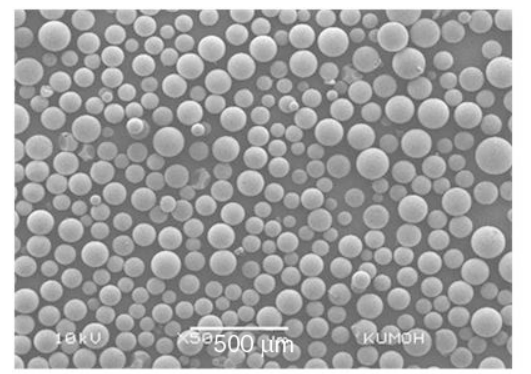

a)

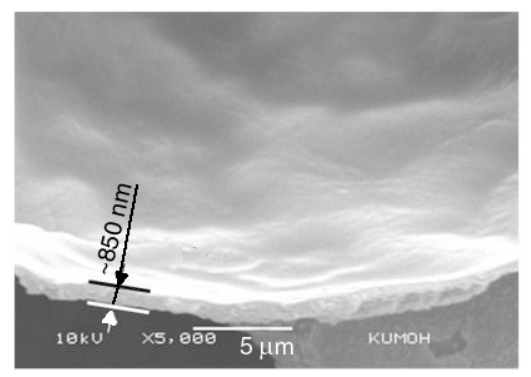

c)

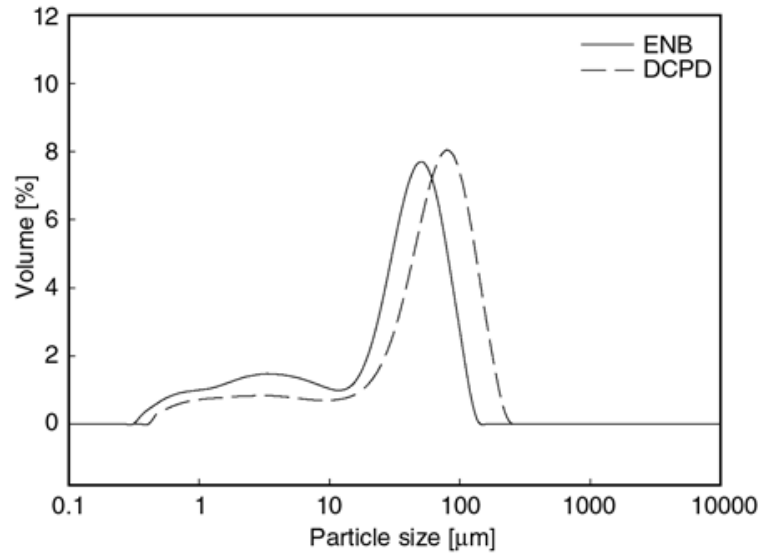

Figure 3. Particle size analysis of endo-DCPD- and ENBmicrocapsules

ters of endo-DCPD- and ENB-microcapsules are found to be $\sim 80$ and $\sim 52 \mu \mathrm{m}$, respectively. A relatively higher viscosity of endo-DCPD than that of ENB produces larger self-healing agent droplets, leading to larger endo-DCPD-microcapsules than ENB-microcapsules in average diameter. The particle size can readily be adjusted by rpm of propeller during dispersion of healing agents in water. The shoulder in the range of $1 \sim 10 \mu$ in this figure may be due to the particles formed by self-coagulation of the wall materials.

\subsection{SEM observations}

Scanning electron microscope images for microcapsules filled with endo-DCPD and ENB were respectively shown in Figures 4 and 5. As noticed from the images in both figures, a perfect sphere

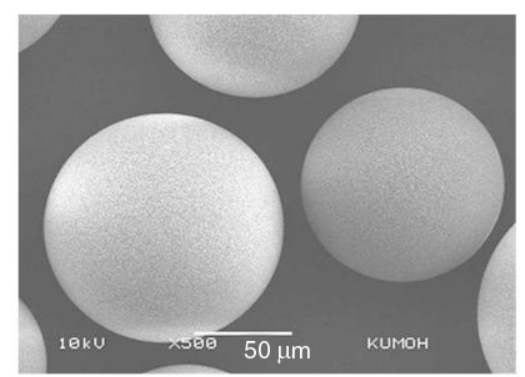

b)

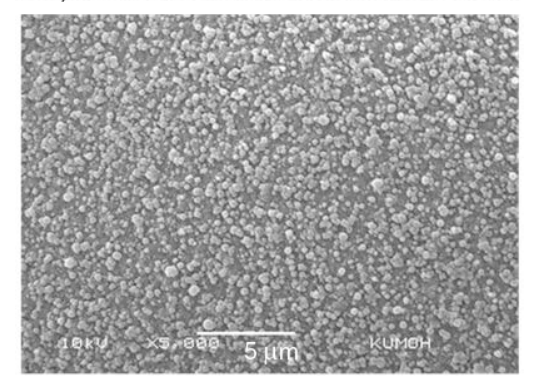

d)

Figure 4. Scanning electron microscopic pictures of endo-DCPD-microcapsules at $\mathrm{rpm}=500$ showing the shape of the microcapsules (a and b), and fractured microcapslues showing inner (c) and outer surface (d) 


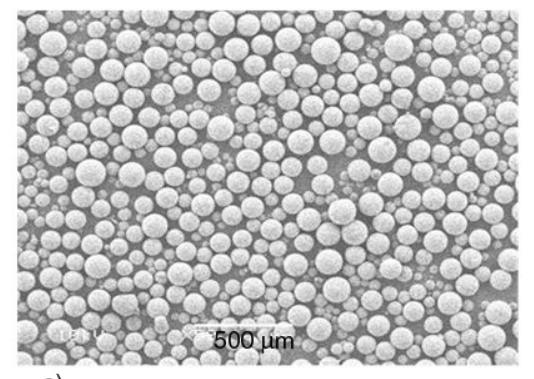

a)

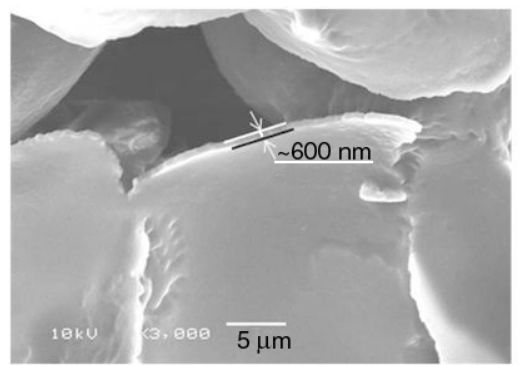

c)

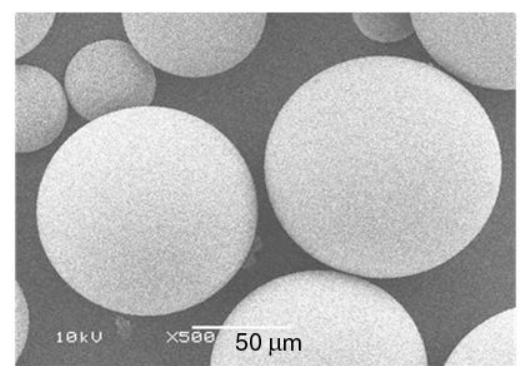

b)

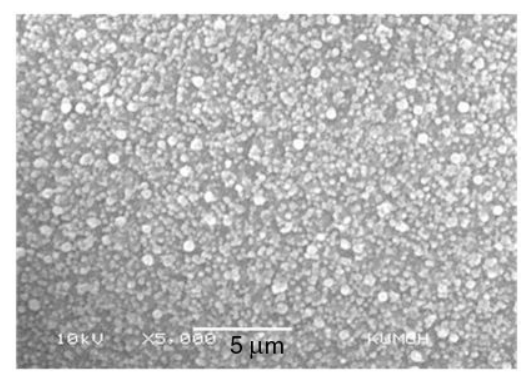

d)

Figure 5. Scanning electron microscopic pictures of ENB-microcapsules at rpm $=500$ showing the shape of the microcapsules (a and b), and fractured microcapslues showing inner (c) and outer surface (d)

with similar inner and outer surfaces was produced. There is no debris for both self-healing agents. Looking at the surface is somewhat rough external surface and smooth inner wall. The rough outer surface of microcapsules is thought to contribute to improving the adhesion between the host matrix and capsules by increasing contact area with the matrix material. The thicknesses of the shells were found to be $\sim 880 \pm 80$ and $\sim 620 \pm 60 \mathrm{~nm}$ from SEM images of endo-DCPD- and ENB-microcapsules, respectively.

\subsection{Fluorescence microscope observations}

In this study, the morphology of microcapsules and the release of the self-healing agent from microcapsules were observed after inducing cracks on an epoxy coating layer dispersed with $5 \mathrm{wt} \%$ of endoDCPD-microcapsules by means of a fluorescence microscope. Figure 6 contains fluorescent microscope images (a), (b), and (c) before damaging and (d), (e), and (f) after damaging taken at different excitation wavelengths of 350,480 , and $546 \mathrm{~nm}$, respectively. The vivid images could be viewed for all excitation wavelengths in different emission colors; blue at $350 \mathrm{~nm}$, green at $480 \mathrm{~nm}$, and red at $546 \mathrm{~nm}$. Note that the image without a fluorescent dye showed completely black in color. The images showed a spherical shape of microcapsules as observed by SEM. It is interesting to notice that the capsule shell is very bright, indicating the incorporation of fluorescent dye into the shell material. Note that the dye was mixed with self-healing agent, followed by dispersion in water and then in-situ polymerization of shell materials reaction at higher temperature. This is presumably due to the migration of dye to shell forming material during the initial stage of reaction. The images (d), (e), and (f) in Figure 6 for cracked coating layer embedded with $5 \mathrm{wt} \%$ of Grubbs catalyst and $5 \mathrm{wt} \%$ of microcapsules show a bright line starting from microcapsules. This is considered to be the trace of transport of self-healing agent between crack planes released from a microcapsule. The brightest fluorescence was observed in the vicinity of the microcapsules where the release of self-healing agent starts to the cracks because the larger amount healing agent is spread to the exit of crack. Also, it is shown that the propagating crack runs around one of small microcapsule at left-top in the image. The black spots shown in the images (d), (e), and (f) are believed to come from the Grubbs catalyst in the matrix. In this work, the addition of a fluorescent dye to self-healing agent enables to observe the track of the self-healing agent release into cracks from microcapsules at three different UV wavelengths. This means that the addition of fluorescent dye to self-healing agent may be useful in studying the details of cracks which are crucial in a microcapsule-based self-healing methodology.

\section{Conclusions}

In this study, microcapsules containing self-healing agents, endo-DCPD and ENB, and a fluorescent 


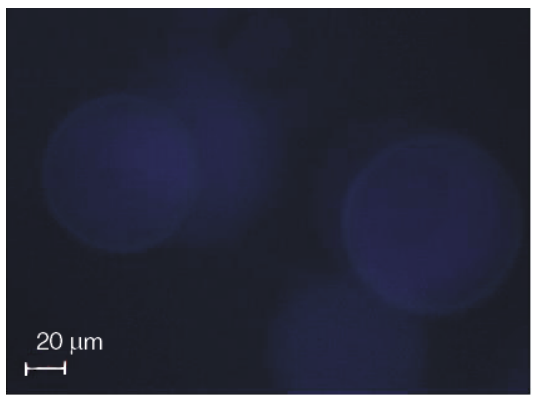

a)

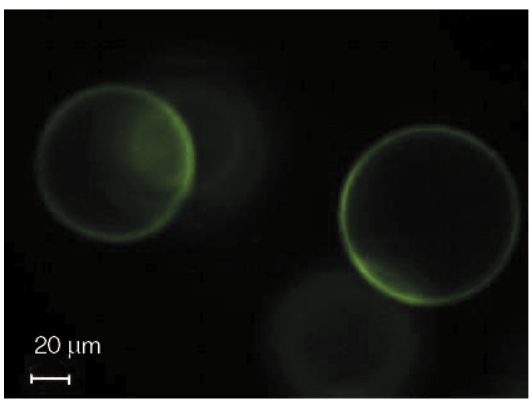

b)

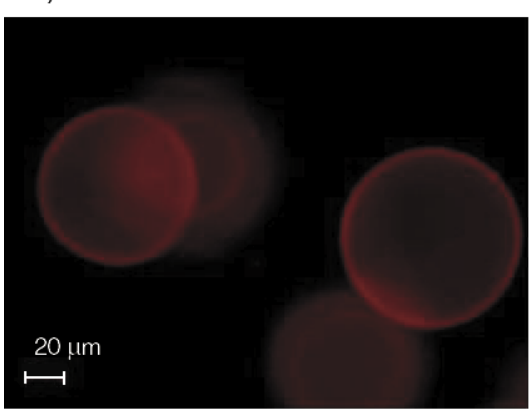

c)

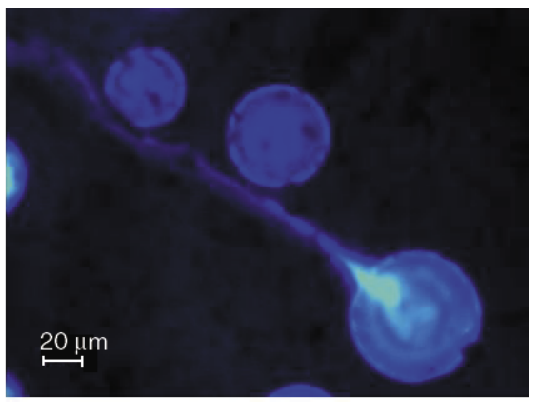

d)

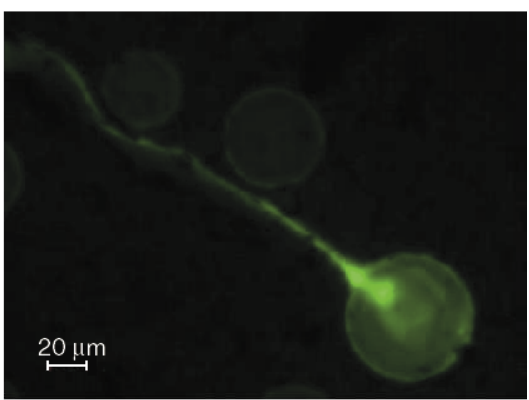

e)

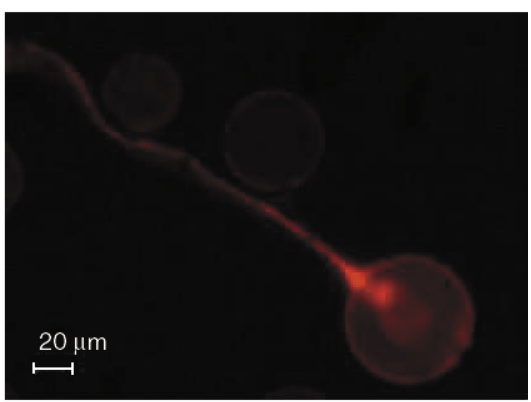

f)

Figure 6. Fluorescent microscope images for a coating layer dispersed with endo-DCPD microcapsules containing a fluorescent dye (before damage, left) and a fluorescent dye in the presence of Grubbs catalyst (after damage, right) at different excited wavelengths; (a) and (d) at $\lambda=350 \mathrm{~nm}$, (b) and (e) $\lambda=480 \mathrm{~nm}$, (c) and (f) $\lambda=546 \mathrm{~nm}$

dye surrounded by the MUF shell were successfully synthesized and analyzed to develop a self-healing coating system. Thermogravimetric analysis showed that endo-DCPD-microcapsules have a significantly higher thermal stability than ENB-microcapsules. From scanning electron microscope observations, both endo-DCPD- and ENB-microcapules have similar morphologies in shape and inner/outer surfaces but different shell thicknesses. The use of a fluorescent dye gave rise to the clear visual images of microcapsules and cracks filled the self-healing agent from ruptured microcapsules after damage at different excited wavelengths.

\section{Acknowledgements}

This research was supported by Basic Science Research Program through the National Research Foundation of Korea (NRF) funded by the Ministry of Education, Science and Technology (2011-0009510).

\section{References}

[1] Kumar A., Stephenson L. D., Murray J. N.: Self-healing coatings for steel. Progress in Organic Coatings, 55, 244-253 (2006).

DOI: 10.1016/j.porgcoat.2005.11.010

[2] Sauvant-Moynot V., Gonzales S., Kittel J.: Self-healing coatings: An alternative route for anticorrosion protection. Progress in Organic Coatings, 63, 307-315 (2008). DOI: $10.1016 /$ j.porgcoat.2008.03.004

[3] Blaiszik B. J., Sottos N. R., White S. R.: Nanocapsules for self-healing materials. Composites Science and Technology, 68, 978-986 (2008).

DOI: 10.1016/j.compscitech.2007.07.021

[4] Cho S. H., White S. R., Braun P. V.: Self-healing polymer coatings. Advanced Materials, 21, 645-649 (2009). DOI: $10.1002 / \mathrm{adma} .200802008$

[5] García S. J., Fischer H. R., van der Zwaag S.: A critical appraisal of the potential of self healing polymeric coatings. Progress in Organic Coatings, 72, 211-221 (2011). DOI: 10.1016/j.porgcoat.2011.06.016 
[6] Samadzadeh M., Hatami Boura S., Peikari M., Kasiriha S. M., Ashrafi A.: A review on self-healing coatings based on micro/nanocapsules. Progress in Organic Coatings, 68, 159-164 (2010).

DOI: 10.1016/j.porgcoat.2010.01.006

[7] Brown E. N., Kessler M. R., Sottos N. R., White S. R.: In situ poly(urea-formaldehyde) microencapsulation of dicyclopentadiene. Journal of Microencapsulation, 20, 719-730 (2003).

DOI: $10.1080 / 0265204031000154160$

[8] Li H., Wang R., Hu H., Liu W.: Surface modification of self-healing poly(urea-formaldehyde) microcapsules using silane-coupling agent. Applied Surface Science, 255, 1894-1900 (2008).

DOI: 10.1016/j.apsusc.2008.06.170

[9] Wang R., Li H., Hu H., He X., Liu W.: Preparation and characterization of self-healing microcapsules with poly(urea-formaldehyde) grafted epoxy functional group shell. Journal of Applied Polymer Science, 113, 1501-1506 (2009).

DOI: $10.1002 /$ app.30001

[10] Yuan L., Liang G-Z., Xie J-Q., He S-B.: Synthesis and characterization of microencapsulated dicyclopentadiene with melamine-formaldehyde resins. Colloid and Polymer Science, 285, 781-791 (2007).

DOI: $10.1007 / \mathrm{s} 00396-006-1621-5$
[11] Liu X., Sheng X., Lee J. K., Kessler M. R.: Synthesis and characterization of melamine-urea-formaldehyde microcapsules containing ENB-based self-healing agents. Macromolecular Materials and Engineering, 294, 389-395 (2009).

DOI: $10.1002 /$ mame.200900015

[12] Liu X., Lee J. K., Kessler M. R.: Microencapsulation of self-healing agents with melamine-urea-formaldehyde by the shirasu porous glass (SPG) emulsification technique. Macromolecular Research, 19, 1056-1061 (2011).

DOI: $10.1007 / \mathrm{s} 13233-011-1009-3$

[13] Mauldin T. C., Kessler M. R.: Self-healing polymers and composites. International Materials Reviews, 55, 317-346 (2010). DOI: $10.1179 / 095066010 X 12646898728408$

[14] Bleay S. M., Loader C. B., Hawyes V. J., Humberstone L., Curtis P. T.: A smart repair system for polymer matrix composites. Composites Part A: Applied Science and Manufacturing, 32, 1767-1776 (2001). DOI: $10.1016 / \mathrm{S} 1359-835 \mathrm{X}(01) 00020-3$

[15] Pang J. W. C., Bond I. P.: A hollow fibre reinforced polymer composite encompassing self-healing and enhanced damage visibility. Composites Science and Technology, 65, 1791-1799 (2005). DOI: $10.1016 /$ j.compscitech.2005.03.008 